\title{
The Automorphism Groups of Steiner Triple Systems Obtained by the Bose Construction
}

\author{
G.J. LOVEGROVE \\ graham.lovegrove@uk.thalesgroup.com; lovegroves@compuserve.com \\ Department of Pure Mathematics, The Open University, Walton Hall, Milton Keynes MK7 6AA, \\ United Kingdom
}

Received February 12, 2001; Revised May 31, 2002; Accepted January 13, 2003

\begin{abstract}
The automorphism group of the Steiner triple system of order $v \equiv 3(\bmod 6)$, obtained from the Bose construction using any Abelian Group $G$ of order $2 s+1$, is determined. The main result is that if $G$ is not isomorphic to $Z_{3}^{n} \times Z_{9}^{m}, n \geq 0, m \geq 0$, the full automorphism group is isomorphic to $\operatorname{Hol}(G) \times Z_{3}$, where $\operatorname{Hol}(G)$ is the Holomorph of $G$. If $G$ is isomorphic to $Z_{3}^{n} \times Z_{9}^{m}$, further automorphisms occur, and these are described in full.
\end{abstract}

Keywords: Steiner triple system, Bose construction, automorphism

\section{Introduction}

A Steiner triple system of order $v, D=\operatorname{STS}(v)$, is an ordered pair $(V, \mathcal{B})$ where $V$ is a set of elements or points, of cardinality $v$, and $\mathcal{B}$ is a collection of 3-element subsets of $V$, called blocks or triples which collectively have the property that every 2-element subset of $V$ is contained in exactly one block. It is well-known that such systems exist if and only if $v \equiv 1$ or $3(\bmod 6)$, a fact first proved by Kirkman in 1847 [3]. Nearly 100 years later, in 1939, Bose [1] gave a particularly elegant construction in the case where $v=6 s+3$. Let $G$ be an Abelian group of order $2 s+1$, which we shall always write additively. It will be convenient to assume that $s \geq 1$, thereby excluding the trivial case of the STS(3).

Take $V=G \times Z_{3}$, and $\mathcal{B}$ to be the collection of blocks:

$$
\begin{aligned}
& \{(x, 0),(x, 1),(x, 2)\}, x \in G, \\
& \{(x, 0),(y, 0),(z, 1)\}, x, y, z \in G, x \neq y, 2 z=x+y, \\
& \{(x, 1),(y, 1),(z, 2)\}, x, y, z \in G, x \neq y, 2 z=x+y, \\
& \{(x, 2),(y, 2),(z, 0)\}, x, y, z \in G, x \neq y, 2 z=x+y .
\end{aligned}
$$

Diagramatically the Bose construction can be represented as follows. 


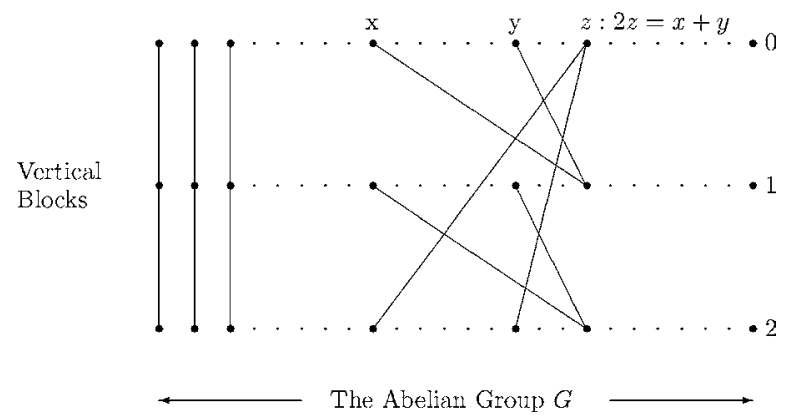

The construction works because in an odd order Abelian group, given any two of the element $x, y, z$, the equation $2 z=x+y$ uniquely determines the third. The Bose construction is capable of various modifications and generalisations; in particular the group $G$ may be replaced by any commutative idempotent Latin square $L$. In this latter scheme, the element $z$ is chosen to be $z=L(x, y)$, the entry in row $x$ and column $y$ of $L$. However in this paper we will be concerned only with the original construction based on an odd order Abelian group as given by Bose.

The purpose of this paper is to determine the full automorphism group of a Steiner triple system, $D$, constructed by the Bose method using an odd order Abelian group $G$. It is clear from the definition of $D$ that, if $B=\{(x, X),(y, Y),(z, Z)\} \in \mathcal{B}$, then $v+B=$ $\{v+(x, X), v+(y, Y), v+(z, Z)\} \in \mathcal{B}$ for any $v \in V$; also, that for any $\beta \in \operatorname{Aut}(G)$,

$$
\beta(B)=\{(\beta(x), X),(\beta(y), Y),(\beta(z), Z)\} \in \mathcal{B}
$$

Thus the bijection $[v, \beta]$ on $V$ defined by

$$
[v, \beta](x, X)=v+(\beta(x), X)
$$

clearly defines an automorphism of $D$.

One of the main results of this paper is that, unless $G$ is a product of copies of $Z_{3}$ and $Z_{9}$, all elements of $\operatorname{Aut}(D)$ are of this form. We shall call the automorphisms of form $B \mapsto v+B$ translations.

Before proceeding, some further terminology and definitions are appropriate. In the Bose construction, there is a natural distinction between two types of block, those of the form $(x, 0),(x, 1),(x, 2)$ and the remainder. We will need to refer to and use this distinction throughout this paper, and therefore, in analogy with the usual diagrammatic representation of the Bose construction as given for example in [5], page 114, the former will be referred to as vertical blocks, and the remainder as non-vertical. Also of importance will be the signature of a block. Defining the label of an element to be the $Z_{3}$ component, the signature of a block is sum of the values of the labels of the block modulo 3. Observe that the signature of a vertical block is zero, the signature of a non-vertical block is 1 , and that no block has signature 2 . 


\section{Block signatures and automorphism type}

In this section we use the concept of block signature to begin to classify the types of automorphism which can exist on a Bose design $D$, and show that two particular types of automorphism exist only in the unique STS(9), the Bose design on $Z_{3}$.

Lemma 2.1 If $E$ is a $3 \times 3$ matrix of points of $D$ such that the rows and columns are all distinct blocks of $D$, then:

(a) all the rows of $E$ have the same signature,

(b) all the columns of $E$ have the same signature,

(c) if all the rows (columns) of $E$ have signature 0, then all the columns (rows) have signature 1.

Proof: Suppose that one row has signature 0 , i.e. it is a vertical block. Then since all rows and columns of $E$ are distinct blocks of $D$, all the columns of $E$ must be non-vertical. Hence the sum of the signatures of the columns is 0 modulo 3 , and so every row has signature 0 . Similarly, if one column has signature 0 , then all rows are non-vertical, and every column has signature 0 . The third possibility is that all rows and columns have signature 1 .

The next two lemmas use this result to classify the automorphisms of $D$ by their actions on the vertical blocks of $D$.

Lemma 2.2 A member of Aut $(D)$ maps the vertical blocks of $D$ either all to vertical blocks or all to non-vertical blocks.

Proof: Let $\phi$ be any member of $\operatorname{Aut}(D)$, and $x, y ; x \neq y$ any two elements of $G$. Apply Lemma 2.1 to the image under $\phi$ of the matrix:

$$
\begin{array}{ccc}
(x, 0) & (x, 1) & (x, 2) \\
(y, 0) & (y, 1) & (y, 2) \\
\left(\frac{x+y}{2}, 1\right) & \left(\frac{x+y}{2}, 2\right) & \left(\frac{x+y}{2}, 0\right)
\end{array}
$$

Suppose that under $\phi$, the top row is mapped to a vertical (non-vertical) block. Then the other rows are mapped similarly.

The above lemma allows the classification of automorphisms into the types vertical and non-vertical, according to whether it maps all vertical blocks to vertical blocks or nonvertical blocks.

We shall say that a vertical block is mapped even/oddly by an automorphism if it is mapped to a vertical block, and the labels $Z_{3}$ are permuted evenly/oddly.

Lemma 2.3 A vertical automorphism of D maps the vertical blocks of $D$ either all evenvertically or all odd-vertically. 
Proof: Let $\phi$ be a vertical automorphism of $D$, and consider the image under $\phi$ of the matrix of Lemma 2.2. Suppose that some row is mapped even-vertically, and another oddvertically. We shall consider only the values of the labels of the individual elements, as defined above, so we can write the values for these two rows as:

$$
\begin{array}{lll}
j & j+1 & j+2 \\
i & i+2 & i+1
\end{array}
$$

Since by Lemma 2.1 all the columns of the image matrix have signature 1, the third row must have label values which are all $1-i-j$, which is not valid for a block of $D$.

Lemmas 2.2 and 2.3 enable us to classify automorphisms of $D$ as even-vertical, oddvertical, or non-vertical according to whether it maps the vertical blocks of $D$ evenvertically, odd-vertically, or non-vertically.

The final result of this section shows that all the automorphisms of all but just one Bose design are even-vertical.

Theorem 2.1 If any element $\phi$ of $\operatorname{Aut}(D)$ is either odd-vertical, or non-vertical, then $G \cong Z_{3}$, and $D$ is the unique $\operatorname{STS}(9)$.

Proof: We prove the result for odd-vertical automorphisms first. The automorphism acts oddly on all vertical blocks. Suppose that $x$ and $y$ are non-zero elements of $G$, and that $x \neq y$ and $x \neq 2 y$. Suppose the label values of the image of the block $\{(0,0),(0,1),(0,2)\}$ are $i, i+2, i+1$. Now consider the image under $\phi$ of the matrix:

$$
\begin{array}{ccc}
(2 y-x, 0) & (x, 0) & (y, 1) \\
(x-2 y, 0) & (-x, 0) & (-y, 1) \\
(0,1) & (0,1) & (0,2)
\end{array}
$$

All columns and the first two rows of this matrix are non-vertical blocks, and by Lemma 2.2 transform to non-vertical blocks. The total of the signatures of the columns is zero. The signatures of the top two rows are both 1 . But the signature of the bottom row is $(i+2)+$ $(i+2)+(i+1)=2$, a contradiction. So one of the initial assumptions must be false.

We now prove the non-vertical case. If $\phi$ is non-vertical, then each vertical block is the image under $\phi$ of a non-vertical block. Since the composition of a non-vertical automorphism with a translation is clearly still non-vertical, we can choose that the block $\{(0,0),(0,1),(0,2)\}$ should be the image of the block $\{(x, 0),(-x, 0),(0,1)\}$ respectively for some non-zero $x \in G$ without affecting the non-vertical property of $\phi$. Then by Lemma 2.1, the image of the matrix:

$$
\begin{array}{lll}
(x, 2) & (-x, 2) & (0,0) \\
(x, 1) & (-x, 1) & (0,2) \\
(x, 0) & (-x, 0) & (0,1)
\end{array}
$$

has vertical blocks as rows and non-vertical blocks as columns. 
Now consider the matrix:

$$
\begin{array}{ccc}
(2 x, 0) & (0,0) & (x, 1) \\
(-2 x, 0) & (0,0) & (-x, 1) \\
(0,1) & (0,0) & (0,2)
\end{array}
$$

The rows of this matrix all map to non-vertical blocks because the image of $(0,0)$ can only be in one vertical block, namely the image of $\{(x, 2)(-x, 2)(0,0)\}$. The last column maps to a vertical block, and the image of the second column has signature zero, so that the first column maps to a block also of signature zero, i.e. vertical. Hence, since $(0,1) \mapsto(0,2)$, $(2 x, 0)$ maps either to $(0,0)$ or $(0,1)$. But $(0,0)$ and $(0,1)$ are the images of $( \pm x, 0)$, so $3 x=0$, since $x$ is non-zero.

Suppose now that $G$ has another non-zero element, $y \neq x$ or $2 x$, and consider the matrix:

$$
\begin{array}{ccc}
\left(\frac{1}{2} y, 0\right) & (0,2) & (y, 2) \\
\left(-\frac{1}{2} y, 0\right) & (0,2) & (-y, 2) \\
(0,1) & (0,2) & (0,0)
\end{array}
$$

Then all the rows map to non-vertical blocks, because the image of $(0,2)$ is only included in one vertical block, namely the image of $\{(x, 1),(-x, 1),(0,2)\}$. In the same way the first and last columns map to non-vertical blocks, because the only vertical block containing the image of the point $(0,1)$ is the image of the block $\{(x, 0),(-x, 0),(0,1)\}$, which is not equal to $\left\{\left(\frac{1}{2} y, 0\right),\left(-\frac{1}{2} y, 0\right),(0,1)\right\}$ by the assumption on $y$, and similarly the only vertical block containing the image of the point $(0,0)$ is the image of the block $\{(x, 2),(-x, 2),(0,0)\}$, which is not equal to $\{(y, 2),(-y, 2),(0,0)\}$. The image of the middle column has signature zero, hence the sum of the row signatures does not equal that of the columns, and the initial assumption must be false.

\section{Standard automorphisms}

In this section we shall be concerned with the way in which automorphisms of a Bose design $D$ permute the labels of vertical blocks. We shall call an automorphism group standard if each of its elements is even-vertical and subjects the labels of every vertical block to the same even permutation (which can equally well be represented as a translation by an element of $Z_{3}$ ). A group of even-vertical automorphisms is non-standard if it is not standard. The distinction is important, because it turns out that for most Bose designs $D$, the full automorphism group is standard. In this section we shall prove that the only groups $G \neq Z_{3}$ for which Aut $D$ is non-standard are products of powers of $Z_{3}$ and/or $Z_{9}$, and will give a simple expression for the structure of Aut $D$ in the standard case.

We shall say that an even-vertical automorphism $\phi$ is standard on an element $x$ of $G$ if $\phi$ permutes the labels of $\{(x, 0),(x, 1),(x, 2)\}$ in the same way as those of $\{(0,0),(0,1)$, $(0,2)\}$. 
Theorem 3.1 If Aut $D$ is non-standard, then all elements of $G$ are of order either 3 or 9 , and so $G$ is isomorphic to a direct sum of copies of $Z_{3}$ and/or $Z_{9}$.

Proof: Note that if $G=0$, then $\operatorname{Aut}(D)$ trivially has no non-standard elements, and we henceforth assume that $|G| \geq 3$. Suppose that $\phi$ is an automorphism of $D$, which acts non-standardly on $x \in G$. Consider the matrix

$$
\begin{array}{ccc}
(0,0) & (0,1) & (0,2) \\
(x, 2) & (x, 0) & (x, 1) \\
(-x, 2) & (-x, 0) & (-x, 1)
\end{array}
$$

Since the composition of $\phi$ with a translation still acts non-standardly on $x$, we may assume that the top row maps to $\{(u, 0),(u, 1),(u, 2)\}$ for some $u \in G$. Then the next row maps to either $\{(v, 0),(v, 1),(v, 2)\}$ or $\{(v, 1),(v, 2),(v, 0)\}$ for some $v \in G$, in which case the third row maps to either $\{(w, 1),(w, 2),(w, 0)\}$ or $\{(w, 0),(w, 1),(w, 2)\}$ respectively for some $w \in G$. There is no loss of generality, because $x$ can be renamed $-x$ or vice-versa, in assuming the former in each case. So the above matrix maps to

$$
\begin{array}{lll}
(u, 0) & (u, 1) & (u, 2) \\
(v, 0) & (v, 1) & (v, 2) \\
(w, 1) & (w, 2) & (w, 0)
\end{array}
$$

Hence $u+v=2 w$. Suppose $3 x \neq 0$. Then, by considering the blocks $\{(3 x, 1),(-x, 1)$, $(x, 2)\}$ and $\{(-3 x, 0),(x, 0),(-x, 1)\}$, it follows that $(3 x, 1)$ maps to $((v+w) / 2,1)$, and $(-3 x, 0)$ maps to $(2 v-w, 0)$. Now from the block $\{(9 x, 0),(-3 x, 0),(3 x, 1)\}$ it is deduced that $(9 x, 0)$ maps to $(2 w-v, 0)=(u, 0)$. Hence $9 x=0$, i.e. all elements of $G$ which are non-standard under $\phi$ have order either 3 or 9 .

We next assert that if $x, y, z \in G$, and $x+y=2 z$, then for any even-vertical automorphism $\phi$ of Aut $D, \phi$ acts standardly either on all three of $x, y, z$, or one exactly of them. This is because $\phi$ preserves signatures, and so $\phi$ either shifts all the labels of any non-vertical block containing $x, y$ and $z$ by the same value, or all by different values. Now suppose that $\phi$ acts non-standardly on $x$ and standardly on $y \neq 0$. Consider the block $\left\{(x, 0),(y, 0),\left(\frac{x+y}{2}, 1\right)\right\}$. Then $\phi$ acts non-standardly on $\frac{x+y}{2}$. Thus $9\left(\frac{x+y}{2}\right)=0$, giving $9 y=0$, i.e. all elements, other than the identity, have order either 3 or 9 .

The full automorphism group of every Bose design except for those designs constructed from products of copies of $Z_{3}$ and/or $Z_{9}$ is therefore standard. The remaining automorphism groups possess standard subgroups. The following result provides the structure for all standard automorphism groups.

Recall that a semidirect product $G \times{ }_{\theta} H$ is formed from two groups $G, H$, and a homomorphism $\theta: H \rightarrow$ Aut $(G)$, with the binary operator $*:\left(g_{1}, h_{1}\right) *\left(g_{2}, h_{2}\right)=\left(g_{1} \theta\left(h_{1}\right)\left(g_{2}\right), h_{1} h_{2}\right)$. If $H \cong \operatorname{Aut}(G)$, with $\theta$ the identity, then the semidirect product is called the Holomorph of $G$, denoted by $\operatorname{Hol}(G)$ (see [4, p. 461]). 
Theorem 3.2 The group of standard automorphisms of $D$ is isomorphic to $\operatorname{Hol}(G) \times Z_{3}$, and so is of order $3|G||\operatorname{Aut}(G)|$.

Proof: If $g \in G, \alpha \in \operatorname{Aut}(G)$, and $a$ is an element of $Z_{3}$, then consider the map [ $\left.g, \alpha, a\right]$ of elements of $D$ defined by: $[g, \alpha, a]:(x, X) \rightarrow(g+\alpha(x), X+a)$. [g, $\alpha, a]$ is clearly a set isomorphism of the elements of $D$. It maps vertical blocks to vertical blocks, and also non-vertical blocks to non-vertical blocks, because if $x, y, z \in G$, and $x+y=2 z$, then $(g+\alpha(x))+(g+\alpha(y))=2 g+\alpha(x+y)=2(g+\alpha(z))$, and because even permutations of the labels preserve blocks. So $[g, \alpha, a]$ is an element of $\operatorname{Aut}(D)$, and is standard. Moreover the mapping $(g, \alpha, a) \mapsto[g, \alpha, a]$ is an injective map of the set $G \times \operatorname{Aut}(G) \times Z_{3} \rightarrow \operatorname{Aut}(D)$, which is also a group homomorphism $\operatorname{Hol}(G) \times Z_{3} \rightarrow \operatorname{Aut}(D)$ because for any element $(x, X)$ of $D$,

$$
\begin{aligned}
{[g, \alpha, a][h, \beta, b](x, X) } & =[g, \alpha, a](h+\beta(x), X) \\
& =(g+\alpha(h)+\alpha \beta(x), X+a+b) \\
& =[g+\alpha(h), \alpha \beta, a+b](x, X)
\end{aligned}
$$

We show that this is a surjection to the subgroup comprising the standard automorphisms of $D$ by constructing a corresponding triple $(g, \alpha, a)$ for any given standard automorphism. Suppose $\phi$ is standard. The element $a \in Z_{3}$ is immediately inferred. Since $\phi$ maps $Z_{3}$ in the same way for every element of $G, \phi$ uniquely defines a map $\phi_{G}: G \rightarrow G$, which is a set isomorphism. $\phi_{G}(0)$ provides the first element of the triple. We need only provide an automorphism of $G$ for the second element. We assert that the map $\Phi: x \mapsto \phi_{G}(x)-\phi_{G}(0)$ is an automorphism of $G$. This follows from the identity:

$$
\phi(\{(x, 0),(y, 0),(z, 1)\})=\left\{\left(\phi_{G}(x), a\right),\left(\phi_{G}(y), a\right),\left(\phi_{G}(z), 1+a\right)\right\}
$$

for mapping of non-vertical blocks, applied to the blocks:

$$
\begin{array}{ccc}
(x, 0) & (-x, 0) & (0,1) \\
(y, 0) & (0,0) & \left(\frac{1}{2} y, 1\right) \\
(x+y, 0) & (-x, 0) & \left(\frac{1}{2} y, 1\right)
\end{array}
$$

which implies the identities:

$$
\begin{aligned}
\phi_{G}(x)+\phi_{G}(-x) & =2 \phi_{G}(0) \\
\phi_{G}(y)+\phi_{G}(0) & =2 \phi_{G}\left(\frac{1}{2} y\right) \\
\phi_{G}(x+y)+\phi_{G}(-x) & =2 \phi_{G}\left(\frac{1}{2} y\right)
\end{aligned}
$$


which implies:

$$
\phi_{G}(x+y)=\phi_{G}(x)+\phi_{G}(y)-\phi_{G}(0),
$$

Therefore $\Phi(x+y)=\Phi(x)+\Phi(y)$, and $\Phi$ is an isomorphism of $G$. Clearly $\left[\phi_{G}(0), \Phi, a\right]=$ $\phi$, and we have the required isomorphism.

\section{Non-standard automorphisms}

As has been proved in the previous section, non-standard automorphisms are only possessed by Bose designs constructed from groups $G$ of the form: $Z_{3}^{n} \times Z_{9}^{m}, n+m \neq 0$. The nonstandard and standard automorphisms of a Bose design together also form an automorphism group, which is the whole automorphism group unless $G=Z_{3}$. We now derive the structure for this group.

Lemma 4.1 If $x, y \in Z_{3}$, then

$$
(-2)^{x}+(-2)^{y} \equiv(-2)^{x+y}+1(\bmod 9)
$$

Proof: There are only nine cases to check, seven of which are trivial.

We can represent any even-vertical automorphism $\phi$ of $D$ as a pair of maps $(\psi, \kappa)$, $\psi: G \rightarrow G, \kappa: G \rightarrow Z_{3}$, where $\psi$ is a set automorphism of $G$, and if $(x, X)$ is any point of $D$,

$$
\phi:(x, X) \mapsto(\psi(x), X+\kappa(x))
$$

The next lemma characterises $\kappa$ as a map of groups, and proves identities necessary for the characterisation of the automorphism group.

Lemma 4.2 If $G$ of the form: $Z_{3}^{n} \times Z_{9}^{m}$, and $\phi=(\psi, \kappa)$ is any even-vertical automorphism of $D$, then the following are true:

(a) If $x, y, z \in G$, and if $x+y=2 z$, then $\kappa(x)+\kappa(y)+\kappa(z) \equiv 0(\bmod 3)$

(b) The map $G \rightarrow Z_{3}$ defined by $u \mapsto \kappa(u)-\kappa(0)$ is a homomorphism.

(c) If $x, y, z \in G$, and $x+y=2 z$, then

$$
(-2)^{\kappa(x)} \psi(x)+(-2)^{\kappa(y)} \psi(y)+(-2)^{1+\kappa(z)} \psi(z)=0
$$

(d) If $x, y, z \in G$, and $x+y=2 z$, then

$$
(-2)^{\kappa(x)}+(-2)^{\kappa(y)}+(-2)^{1+\kappa(z)} \equiv 0(\bmod 9)
$$

\section{Proof:}

(a) A non-vertical block $\{(x, 0),(y, 0),(z, 1)\}$ will be mapped to a non-vertical block with label values $\kappa(x), \kappa(y)$, and $1+\kappa(z)$ respectively. Since $\phi$ is a vertical automorphism, 
it preserves the signatures of blocks, so $\kappa(x)+\kappa(y)+1+\kappa(z) \equiv 1(\bmod 3)$. The result is also trivially true if $x=y=z$.

(b) We have to show that for any $u, v \in G, \kappa(u+v)-\kappa(0)=\kappa(u)-\kappa(0)+\kappa(v)-\kappa(0)$, i.e. $\kappa(u+v)+\kappa(0)=\kappa(u)+\kappa(v)$. But, putting $u+v=2 w$, we have from (a) by writing $u+v=2 w=(u+v)+0$,

$$
\kappa(u)+\kappa(v)+\kappa(w)=0=\kappa(u+v)+\kappa(0)+\kappa(w)
$$

and the result follows.

(c) Observe from (a) that $\kappa(x), \kappa(y), \kappa(z)$ are either all the same or all different. Consider the mapping of the block $\{(x, X),(y, X),(z, X+1)\}$ by $(\psi, \kappa)$ to the block $\{\psi(x), X+$ $\kappa(x)),(\psi(y), X+\kappa(y)),(\psi(z), X+1+\kappa(z))\}$. If $\kappa(x), \kappa(y), \kappa(z)$ are all the same, then $\psi(x)+\psi(y)=2 \psi(z)$, and hence $\psi(x)+\psi(y)+(-2) \psi(z)=0$. If they are all different, then suppose without loss of generality that $\kappa(x)=1+\kappa(z)$, and $\kappa(y)=2+\kappa(z)$, then the image of $\{(x, X),(y, X),(z, X+1)\}$ is

$$
\{(\psi(x), X+1+\kappa(z)),(\psi(y), X+2+\kappa(z)),(\psi(z), X+1+\kappa(z))\}
$$

and so $\psi(x)+\psi(z)=2 \psi(y)$, or $\psi(x)+(-2) \psi(y)+\psi(z)=0$, so

$$
(-2)^{1+\kappa(z)} \psi(x)+(-2)^{2+\kappa(z)} \psi(y)+(-2)^{1+\kappa(z)} \psi(z)=0
$$

and the required result follows.

(d) It is only necessary to use (a), and to check the three cases where $\kappa(x), \kappa(y), \kappa(z)$ are: all the same, an even permutation of $(0,1,2)$, and an odd permutation of the same.

The previous two lemmas provide the material to identify the group of all even-vertical automorphisms of $D$.

Theorem 4.1 If $\phi=(\psi, \kappa)$ is an even-vertical automorphism of $D$, then the map $x \mapsto$ $(-2)^{\kappa(x)}(\psi(x)-\psi(0))$ is an automorphism of $G$. The group of even-vertical automorphisms of $D$ is isomorphic to a group on the set $Z_{3} \times G \times \operatorname{Hom}\left(G, Z_{3}\right) \times \operatorname{Aut}(G)$, by the map:

$$
(\kappa, \psi)(x) \mapsto\left(\kappa(0), \psi(0), \kappa(x)-\kappa(0),(-2)^{\kappa(x)}(\psi(x)-\psi(0))\right)
$$

so that all even-vertical automorphisms $\phi=(\psi, \kappa)$ of $D$ can be expressed in the form:

$$
\begin{aligned}
& \kappa(x)=a+h(x), \psi(x)=g+(-2)^{-\kappa(x)} \alpha(x) \\
& a \in Z_{3}, h \in \operatorname{Hom}\left(G, Z_{3}\right), g \in G, \alpha \in \operatorname{Aut}(G)
\end{aligned}
$$

Proof: That the map $x \mapsto(-2)^{\kappa(x)}(\psi(x)-\psi(0))$ is a group homomorphism uses Lemma 4.2 parts (c) and (d). Writing $\alpha(x)=(-2)^{\kappa(x)}(\psi(x)-\psi(0))$, (c) and (d) imply 
that if $x+y=2 z$ (including $x=y=z$ ), then:

$$
\begin{aligned}
\alpha(x)+\alpha(y)-2 \alpha(z)= & (-2)^{\kappa(x)} \psi(x)+(-2)^{\kappa(y)} \psi(y)+(-2)^{1+\kappa(z)} \psi(z) \\
& -\psi(0)\left((-2)^{\kappa(x)}+(-2)^{\kappa(y)}+(-2)^{1+\kappa(z)}\right) \\
= & 0
\end{aligned}
$$

So, in the same way as for Lemma 3.2, we can write:

$$
\begin{aligned}
\alpha(2 x)+\alpha(2 y) & =2 \alpha(x+y) \\
2 \alpha(x) & =\alpha(2 x)+\alpha(0) \\
2 \alpha(y) & =\alpha(2 y)+\alpha(0)
\end{aligned}
$$

Since $\alpha(0)=0$, the sum of these three equations yields $\alpha(x)+\alpha(y)=\alpha(x+y)$ for all $x, y \in G$, and $\alpha$ is a group homomorphism on $G$. To show that $\alpha$ is $1-1$ it is sufficient to show that $\alpha(x)=0$ iff $x=0$, since $\alpha$ is a group homomorphism. However from its definition, $\alpha(x)=0$ iff $\psi(x)=\psi(0)$, so $x=0$ since $\psi$ is a set automorphism on $G$.

The above establishes the mapping from $\operatorname{Aut}(D)$ to $Z_{3} \times G \times \operatorname{Hom}\left(G, Z_{3}\right) \times \operatorname{Aut}(G)$ as a map of sets. The map is 1-1 because the first three components of the image determine $\psi(0)$ and $\kappa$, whilst these and the fourth component are sufficient to determine $\psi$.

We next show that the map is onto. We shall show that if $a \in Z_{3}, h \in \operatorname{Hom}\left(G, Z_{3}\right)$, $\alpha \in \operatorname{Aut}(G)$, and $g \in G$, then $(\psi, \kappa)$, where $\kappa: G \mapsto Z_{3}$ is defined as $\kappa(x)=a+h(x)$, and $\psi: G \mapsto G$ is defined as $\psi(x)=g+(-2)^{-\kappa(x)} \alpha(x)$, is an automorphism of $D$. Firstly we assert that $(\psi, \kappa)$ maps blocks to blocks. Clearly it maps vertical blocks to vertical blocks, so in the following we have only to consider non-vertical blocks.

Observe that if $x, y, z \in G$ such that $x+y=2 z$, then

$$
\kappa(x)+\kappa(y)+\kappa(z)=0
$$

since the left-hand side is equal to:

$$
3 a+h(x)+h(y)+h(z)=h(x+y)-2 h(z)=h(x+y-2 z)=0
$$

Also,

$$
(-2)^{\kappa(x)} \psi(x)+(-2)^{\kappa(y)} \psi(y)+(-2)^{1+\kappa(z)} \psi(z)=0
$$

since the left-hand side is equal to:

$$
\begin{aligned}
& =\alpha(x)+\alpha(y)-2 \alpha(z)+\left((-2)^{\kappa(x)}+(-2)^{\kappa(y)}+(-2)^{1+\kappa(z)}\right) g \\
& =0+\left(1+(-2)^{\kappa(x)+\kappa(y)}+(-2)^{1+\kappa(z)}\right) g \\
& =\left(2+(-2)^{\kappa(x)+\kappa(y)+1+\kappa(z)}\right) g \\
& =\left(2+(-2)^{3 a+3 h(z)+1}\right) g=0
\end{aligned}
$$

since $\alpha \in \operatorname{Aut}(G)$ and $h \in \operatorname{Hom}\left(G, Z_{3}\right)$, and by Lemma 4.1. 
The first of these relationships once again shows that $\kappa(x), \kappa(y), \kappa(z)$ are either all the same or all different. If they are all the same, the second relationship gives $\psi(x)+\psi(y)=$ $2 \psi(z)$, and so $(\psi, \kappa)$ maps the block $\{(x, X),(y, X),(z, X+1)\}$ to the block

$$
\{(\psi(x), X+\kappa(x)),(\psi(y), X+\kappa(x)),(\psi(z), 1+X+\kappa(x))\}
$$

If $\kappa(x), \kappa(y), \kappa(z)$ are all different, then $1+\kappa(z)$ is equal either to $\kappa(x)$ or to $\kappa(y)$. Without loss of generality suppose the former. Then $\kappa(y)=1+\kappa(x)$, and the second of the relationships proved above implies

$$
(-2)^{\kappa(x)} \psi(x)+(-2)^{1+\kappa(x)} \psi(y)+(-2)^{\kappa(x)} \psi(z)=0
$$

Hence $\psi(x)+\psi(z)+(-2) \psi(y)=0$ since $(-2)^{\kappa(x)} \neq 0$, so $\psi(x)+\psi(z)=2 \psi(y)$, and $(\psi, \kappa)$ maps the block $\{(x, X),(y, X),(z, X+1)\}$ to the block

$$
\{(\psi(x), X+\kappa(x)),(\psi(z), X+\kappa(x)),(\psi(y), 1+X+\kappa(x))\}
$$

It follows that $(\psi, \kappa)$ maps blocks to blocks.

Secondly we show that $(\psi, \kappa)$ is $1-1$. This follows because if $(\psi, \kappa)(x, X)=(\psi, \kappa)(y, Y)$, then $(-2)^{-\kappa(x)} \alpha(x)+g=(-2)^{-\kappa(y)} \alpha(y)+g$. Since $(-2)$ factors commute with $\alpha$, $\alpha\left((-2)^{-\kappa(x)} x\right)=\alpha\left((-2)^{-\kappa(y)} y\right)$, so $(-2)^{-\kappa(x)+\kappa(y)} x-y=(-2)^{-h(x-y)} x-y=0$ since $\alpha$ is $1-1$. However $h\left((-2)^{z} x\right)=(-2)^{z} h(x)=h(x)$ for any $z \in Z_{3}$, since (-2) is the identity on $Z_{3}$, so

$$
h(x-y)=h\left((-2)^{-h(x-y)} x-y\right)=h(0)=0
$$

and so $x=y$ and $X=Y$, hence $(\psi, \kappa)$ is $1-1$. Thus $(\psi, \kappa)$ is an automorphism of $D$.

This group on the set $Z_{3} \times G \times \operatorname{Hom}\left(G, Z_{3}\right) \times \operatorname{Aut}(G)$ is of order $3^{2 n+3 m+1}|\operatorname{Aut} G|$ for $G=Z_{3}^{n} \times Z_{9}^{m}$, and is the full automorphism group except for the special case $G=Z_{3}$, due to the extra symmetry between $G$ and the label set $Z_{3}$. It is readily shown that the full automorphism group of $\operatorname{STS}(9)$ is isomorphic to $\operatorname{Hol}\left(Z_{3} \times Z_{3}\right)$. However, this is a special case of the final result:

Theorem 4.2 Let $D_{n}$ be the STS of order $3^{n}$ formed on the elements of the group $G=$ $\left(Z_{3}\right)^{n}$ consisting of the direct product of $n$ copies of $Z_{3}$, where the blocks are all triples $\{x, y, z\}, x \neq y \neq z$ such that $x+y+z=0$. Then the full automorphism group of $D_{n}$ is isomorphic to $\operatorname{Hol}(G)$.

Proof: The proof is very similar to that of Theorem 3.2.

The design $D_{n}$ is of course the affine geometry $\operatorname{AG}(n, 3)$.

As an illustration of the results of this and the previous section, we will consider four $\operatorname{STS}(81)$ s, and calculate the sizes of their automorphism groups.

Let $G_{1}=Z_{27}, G_{2}=Z_{3} \times Z_{3} \times Z_{3}, G_{3}=Z_{3} \times Z_{9}$, and $G_{4}=Z_{3} \times Z_{3} \times Z_{3} \times Z_{3}$. Then the Bose constructions on $G_{1}, G_{2}$, and $G_{3}$ are all of order 81 , as is the affine geometry on $G_{4}$. 
Firstly, however, we need to know $|\operatorname{Aut}(G)|$ for $G=Z_{3}^{n}$, and $G=Z_{3} \times Z_{9}$. For the former, the result is well-known, see for example [2, p. 128], as the order of $G L\left(n, Z_{3}\right)$ :

$$
|\operatorname{Aut}(G)|=\prod_{i=0}^{i=n-1}\left(3^{n}-3^{i}\right)
$$

For $G_{3}=Z_{3} \times Z_{9}, \operatorname{Aut}(G)$ is readily seen to be the group of all $2 \times 2$ matrices of form:

$$
\left[\begin{array}{cc}
a & c \\
3 b & d
\end{array}\right]
$$

where $a, b, c \in Z_{3}$, and $d \in Z_{9}$, and the determinant is non-zero modulo 3 . The order of this group is 108 .

Consider first the Bose design on $G_{1}$. By Theorem 3, the automorphism group of this design is standard, since the group has an element of order 27. The order of the automorphism group of the design is then

$$
3\left|Z_{27}\right|\left|\operatorname{Aut}\left(Z_{27}\right)\right|=3 \cdot 27 \cdot 18=1458 .
$$

The design on $G_{2}$ has an non-standard automorphism group of order

$$
3^{7}\left|\operatorname{Aut}\left(G_{2}\right)\right|=3^{7}\left(3^{3}-1\right)\left(3^{3}-3\right)\left(3^{3}-9\right)=2187 \cdot 26 \cdot 24 \cdot 18=24,564,384 .
$$

The design on $G_{3}$ also has an non-standard automorphism group of order

$$
3^{6}\left|\operatorname{Aut}\left(G_{3}\right)\right|=3^{6} \cdot 108=78,732 .
$$

Finally, the design on $G_{4}$, being the affine geometry $\operatorname{AG}(4,3)$, has by 4.2 the automorphism group $\operatorname{Hol}\left(G_{4}\right)$, which has order

$$
\left|G_{4}\right| \cdot\left|\operatorname{Aut}\left(G_{4}\right)\right|=3^{4} \cdot\left(3^{4}-1\right) \cdot\left(3^{4}-3\right) \cdot\left(3^{4}-3^{2}\right) \cdot\left(3^{4}-3^{3}\right)=1,965,150,720
$$

\section{Acknowledgment}

The author would like to thank Professor M.J. Grannell, Professor T.S. Griggs, Dr. F. Holroyd, and Dr. K.A.S. Quinn of the Open University for their kind and helpful advice while writing this paper, and also his employer, Thales Defence Ltd., for financial support.

\section{References}

1. R.C. Bose, "On the construction of balanced incomplete block designs," Ann. Eugenics 9 (1939), 353-399.

2. P.J. Cameron, Combinatorics, Cambridge University Press, Cambridge, England, 1994.

3. T.P. Kirkman, "On a problem in combinations," Cambridge and Dublin Math. Journal 2 (1847), 191-204.

4. S. MacLane and G. Birkhoff, Algebra, MacMillan, London, 1967.

5. A.P. Street and D.J. Street, Combinatorics of Experimental Design, Clarendon Press, Oxford, 1987. 